\title{
Cavities Identification from Partially Overdetermined Boundary Data in Linear Elasticity
}

Jaiem $E^{*}$, Abda AB and Rjaibi $B$

Lamsin Enit Department, University of Tunis El Manar, BP 37, 1002, Tunis, Tunisia

\begin{abstract}
Our main interest in this work is an analysis of geometrical inverse problem related to the detection of cavities, in elasticity framework from partially overdetermined boundary data in two spatial dimensions. For the reconstruction, we have only access to the displacement field and to the normal component of the normal stress. We propose an identification method based on the Kohn-Vogelius formulation combined with the topological gradient method. An asymptotic expansion for an energy function is derived with respect to the creation of a small hole. A one-shot reconstruction algorithm based on the topological sensitivity analysis is implemented. Some numerical experiments concerning the cavities identification are finally reported, highlighting the ability of the method to identify multiple cavities.
\end{abstract}

Keywords: Geometrical inverse problems; Cavities identification; Linear elasticity; Partially overdetermined boundary data; Topological derivative; Asymptotic expansion

\section{Introduction}

Shape reconstruction of cavities (namely holes) is a challenging subject with application to diverse areas such as exploration geophysics, medical imaging and non-destructive testing. In industrial framework for example, it is essential for engineers to check the structure integrity and to establish the behavior of mechanical components before critical damage occurs $[1,2]$.

To such identification tasks (namely to deduce the properties of the hidden interior), boundary data are required. In the context of geometrical inverse problem, it is a question about overdetermined boundary data, namely data provided by measurements distributed on the exterior boundary of the domain of interest $[3,4]$. To the author's best knowledge, all geometric inverse problems in linear elasticity, investigated in the literature, have in common to be defined by complete overdetermined boundary data [3-6] with the exception of a recent work [7], where data appear to be partial.

Driven by the needs from applications in both industry and other sciences, we consider in the present work, the same geometrical inverse problem of recovering cavities in elasticity framework, investigated in [7]. The problem is compounded by missing data which is the main motivation. Indeed, the displacement field and the normal component of the normal stress are accessible whereas no information is given on the shear stress.

The problem under consideration might be formulated as follows: Let $\Omega \subset^{2} \mathbb{R}$ denotes an open and bounded domain with boundary $\Upsilon$ occupied by a linear elastic material, the medium being assumed to be homogeneous and isotropic. Given the normal component of the normal stress imposed $\mathrm{g}$ and the displacement field $f$ measured on the boundary $\Upsilon$

$$
\int \sigma(u) n_{\Upsilon} \cdot n_{\Upsilon}=g \quad \text { on } \Upsilon,
$$

namely the exterior boundary measurements, the linear elasticity inverse problem consists in finding the boundary $\Gamma$ of a bounded domain $\overline{\mathcal{O}} \subset \Omega$ and the displacement field $u$ satisfying

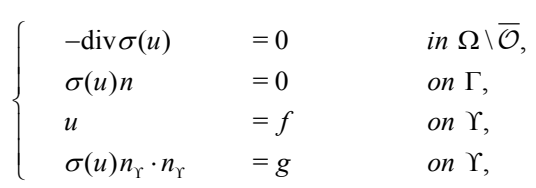

Where $n_{\Upsilon}$ and $n$ are the outward unit normals to the boundary of $\Omega \backslash \overline{\mathcal{O}}$. The stress tensor $\sigma$ and the strain tensor $\varepsilon$ are given by

$$
\begin{aligned}
& \sigma_{i j}(u)=\lambda \operatorname{div}(u) \delta_{i j}+2 \mu \varepsilon_{i j}(u) \\
& \text { and } \varepsilon_{i j}(u)=\frac{1}{2}\left(\frac{\partial u_{i}}{\partial x_{j}}+\frac{\partial u_{j}}{\partial x_{i}}\right), \quad 1 \leq i, j \leq 2
\end{aligned}
$$

Above, $\delta_{i j}$ is the Kronecker symbol and $\lambda$, $\mu$ are the Lamé coefficients related to Young's modulus $E$ and Poisson's ratio $v$ via

$$
\mu=\frac{E}{2(1+v)} \text { and } \lambda=\frac{E v}{(1-2 v)(1+v)} \text {. }
$$

In order to solve our geometrical inverse problem (1), we propose a Dirichlet-Neumann approach by the means of a self regularization technique, namely the Kohn-Vogelius formulation [3,4,7,8]. Indeed,

\begin{tabular}{|c|c|c|}
\hline$-\operatorname{div} \sigma\left(u^{D}\right)$ & $=0$ & in $\Omega \backslash \overline{\mathcal{O}}$ \\
\hline$\sigma\left(u^{D}\right) n$ & $=0$ & on $\Gamma$, \\
\hline$u^{D}$ & $=f$ & on $\Upsilon$ \\
\hline
\end{tabular}
it leads to define two forward problems. One of them is related to the Dirichlet data (the displacement field $f$ ) (2) and the other one is associated to the "Neumann" data (the normal component of the normal stress g) (3)

and

*Corresponding author: Jaiem E, Lamsin Enit Department, University of Tunis El Manar, BP 37, 1002, Tunis, Tunisia, Tel: +21671871022; E-mail: emna23jaiem@gmail.com

Received February 18, 2016; Accepted March 16, 2016; Published March 22 2016

Citation: Jaiem E, Abda AB, Rjaibi B (2016) Cavities Identification from Partially Overdetermined Boundary Data in Linear Elasticity. J Appl Computat Math 5: 295. doi:10.4172/2168-9679.1000295

Copyright: ( 2016 Jaiem E, et al. This is an open-access article distributed under the terms of the Creative Commons Attribution License, which permits unrestricted use, distribution, and reproduction in any medium, provided the original author and source are credited. 


\begin{tabular}{|c|c|c|}
\hline$-\operatorname{div} \sigma\left(u^{N}\right)$ & $=0$ & in $\Omega \backslash \overline{\mathcal{O}}$ \\
\hline$\sigma\left(u^{N}\right) n$ & $=0$ & on $\Gamma$, \\
\hline $\begin{array}{l}\sigma\left(u^{N}\right) n_{\Upsilon} \cdot n_{\Upsilon} \\
u^{N} \cdot \tau\end{array}$ & $\begin{array}{l}=g \\
=f \cdot \tau\end{array}$ & $\begin{array}{l}\text { on } \Upsilon \text {, } \\
\text { on } \Upsilon \text {. }\end{array}$ \\
\hline
\end{tabular}

The main idea of the approach proposed relies on the use of a Kohn-Vogelius functional defined by

$$
\mathcal{J}\left(u^{D}, u^{N}\right):=\frac{1}{2} \int_{\Omega \backslash \overline{\mathcal{O}}}\left(\sigma\left(u^{D}\right)-\sigma\left(u^{N}\right)\right):\left(\varepsilon\left(u^{D}\right)-\varepsilon\left(u^{N}\right)\right)
$$

as it will be explained below. Thus, the geometrical inverse problem (1) is formulated as a topology optimization one

$$
\min _{\overline{\mathcal{O}} \subset \Omega} \mathcal{J}\left(u^{D}, u^{N}\right) .
$$

The important point to note here is that the same geometric inverse problem was addressed in [7] by means of the same Kohn-Vogelius misfit functional (4) combined with the shape gradient method. The theoretical question related to the identifiability was also proved in the case of monotonous cavities [7].

A logical sequel of the work [7] is to combine the shape gradient method with the level set one which is one of promising techniques that can be designed to such shape reconstruction problem $[3,4,8]$. However, one can remark that although the level set method is able to automatically handle topology changes and reconstruct the precise geometry of the cavities $[3,4,8]$, it requires an iterative procedure where at each iteration step, one needs to solve a sequence of forward solutions. As a consequence, the level set method seems to be a slow and expensive process. To overcome this difficulty when solving our geometrical inverse problem, we resort, in this paper, to a one iteration algorithm based on the topological gradient method. It is a recent numerical method of shape and topology optimization of structures, introduced by A. Schumacher in 1995 [9] in the context of compliance minimization in linear elasticity and which makes possible topology changes. Indeed, unlike the classical shape optimization, the topology of the domain may change during the optimization process. A change in topology means removing a small ball from the domain of integration. Our aim is so to find an optimal shape without any a priori assumption about its topology.

The rest of this paper is organized as follows: in the next section, we give a brief review of the topological gradient method and then present its application to the cavities localization problem in the third section. The main result in this part is providing the topological asymptotic expansion by the means of a generalized adjoint method, previously recalled in the second section, and a domain truncation. The fourth section is devoted to numerical implementation using one-iteration algorithm. Indeed, we explore the efficiency of the proposed method by several numerical experiments. The last section contains some comments.

\section{The Topological Gradient Method}

As it does not impose restriction on the topology of the domain, the topological gradient method has been widely applied in literature to different and broad cases of problems as the elasticity framework $[10,11]$, the Stokes system $[12,13]$, the Helmholtz equation [14], the image processing problems $[15,16]$ and many others. We refer to $[13,17,18]$ for a concise overview of the method.

Let us present the basic idea of this approach. We consider a variable, open and bounded domain $\Omega$ of ${ }^{2} \mathbb{R}$ and a cost functional $j()=\mathbb{J}\left(u_{\Omega}\right)$ to be minimized, where $u_{\Omega}$ is solution to a given $\operatorname{PDE}$ defined over $\Omega$. For a small parameter $\rho>0$, let $\Omega=\Omega \backslash\left(\overline{x_{0}+\rho \omega}\right)$ be the perturbed domain obtained by removing a small part $\omega_{\rho}=x_{0}+\rho \omega$ from $\Omega$, where $x_{0} \in \Omega$ and $\omega \subset^{2} \mathbb{R}$ is a fixed open and bounded subset containing the origin, whose boundary $\partial \omega$ is connected and piecewise of class $C^{1}$ (Figure 1).

If $\rho=0$ then $\omega=\varnothing$. Then, a so-called topological asymptotic expansion of the function $j$ is provided by the topological sensitivity theory in the following form

$$
\begin{aligned}
& j\left(\Omega_{\rho}\right)=j(\Omega)+f(\rho) g\left(x_{0}\right)+o(f(\rho)), \\
& \text { where } \lim _{\rho \rightarrow 0} f(\rho)=0 \text { and } f(\rho)>0
\end{aligned}
$$

In other words, the purpose is to study the variations of the objective function $j\left(\Omega_{\rho}\right)$ as $\rho \rightarrow 0$. The topological sensitivity $g\left(x_{0}\right)$, also called the topological gradient can be used like a descent direction in an optimization process. Indeed, to minimize the criterion $j$, one has to create holes at some points where the topological gradient $g$ is negative. $g\left(x_{0}\right)$ is usually computed using the solution of direct and adjoint problems defined on the initial domain and it will be the case in our problem as it will be demonstrated in the forthcoming sections.

\section{A generalized adjoint method}

The following generalized adjoint method $[10,14]$ is applied to the above problem.

Let $\mathbb{V}$ be a fixed Hilbert space. For $\rho \geq 0$, let $a_{\rho}$ be a bilinear, symmetric, uniformly continuous and coercive form on $\mathbb{V}$ and let $l$ be a linear and uniformly continuous form on $\mathbb{V}$. We assume that there exist a bilinear and continuous form $\delta_{a}$, a linear and continuous form $\delta_{l}$ and a real function $f()>0$ defined on $\mathbb{R}_{+}$such that

$$
\begin{aligned}
& \lim _{\rho \rightarrow 0} f(\rho)=0, \\
& \left\|a_{\rho}-a_{0}-f(\rho) \delta_{a}\right\|_{\mathcal{L}_{2}(\mathcal{V})}=o(f(\rho)), \\
& \left\|l_{\rho}-l_{0}-f(\rho) \delta_{l}\right\|_{\mathcal{L}(\mathcal{V})}=o(f(\rho)),
\end{aligned}
$$

where $\mathbb{L}()$ (respectively, $\mathbb{L}(\mathbb{V})$ ) denotes the space of continuous and linear (respectively bilinear) forms on $\mathbb{V}$.

These assumptions will be satisfied in the topology optimization context in the next section. For $\rho \geq 0$, let $u_{\rho}$ be the unique solution [10] to the following problem:

$a_{\rho}\left(u_{\rho}, v\right)=l_{\rho}(v) \quad \forall v \in \mathcal{V}$.

Now, let us consider a function $j\left(\Omega_{\rho}\right)=\mathbb{J}_{\rho}(u)$, where ${ }_{0} \mathbb{J}$ is differentiable with respect to $u$, its derivative being denoted by $D \mathbb{J}(u)$. Moreover, we suppose that there exists a function defined on $\mathbb{V}$ such that

$\mathcal{J}_{\rho}(v)-\mathcal{J}_{0}(u)=D \mathcal{J}(u)(v-u)+f(\rho) \delta_{\mathcal{J}}(u)+o(\|v-u\|+f(\rho)) \quad \forall u, v \in \mathcal{V}$.

For $\rho>0$, we define the Lagrangian operator $\mathbb{L}_{\rho}$ by
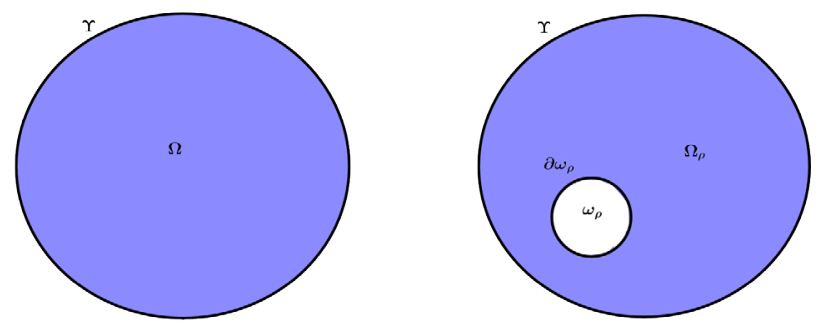

Figure 1: The initial domain and the same domain after the inclusion of the hole. 


$$
\mathcal{L}_{\rho}(u, v)=\mathcal{J}_{\rho}(u)+a_{\rho}(u, v)-l_{\rho}(v) \quad \forall u, v \in \mathcal{V} .
$$

Its variation with respect to $\rho$ is given by

$$
\delta_{\mathcal{L}}(u, v)=\delta_{\mathcal{J}}(u)+\delta_{a}(u, v)-\delta_{l}(v) \quad \forall u, v \in \mathcal{V}
$$

and we have

$$
\mathcal{L}_{\rho}(u, v)-\mathcal{L}_{0}(u, v)=f(\rho) \delta_{\mathcal{L}}(u, v)+o(f(\rho)) \quad \forall u, v \in \mathcal{V} .
$$

Theorem 1: The function $j$ has the asymptotic expansion [10].

$$
j\left(\Omega_{\rho}\right)=j(\Omega)+f(\rho) \delta_{\mathcal{L}}\left(u_{0}, v_{0}\right)+o(f(\rho)),
$$

Where $u_{0}$ is the solution to the problem (6) with $\rho=0$ and $v_{0}$ is the solution to the adjoint problem: find $v_{0} \in \mathcal{V}$ such that

$$
a_{0}\left(w, v_{0}\right)=-D \mathcal{J}\left(u_{0}\right) w \quad \forall w \in \mathcal{V} .
$$

\section{Application to Cavities Identification}

Let $\Omega$ be defined in the same way as in the introduction. The linear elasticity Dirichlet respectively "Neumann" problems, defined on the whole domain $\Omega$, are the following: Find $u_{0}^{D}$ respectively $u_{0}^{N}$ solution of

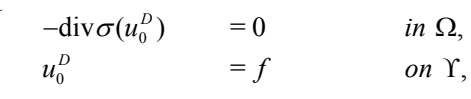

respectively

$$
\begin{aligned}
& -\operatorname{div} \sigma\left(u_{0}^{N}\right) \quad=0 \quad \text { in } \Omega \\
& \sigma\left(u_{0}^{N}\right) n_{\Upsilon} \cdot n_{\Upsilon} \quad=g \quad \text { on } \Upsilon, \\
& u_{0}^{N} \cdot \tau \quad=f \cdot \tau \quad \text { on } \Upsilon \text {, }
\end{aligned}
$$

Where $\sigma, \Upsilon, n_{\Upsilon}$ and $\tau$ are as already defined in the first section. Let us also consider $\Omega_{\rho}$ as previously specified in the previous section and the displacements $u_{\Omega}^{D}$ and $u_{\Omega}^{N}$ defined as the solutions of the following Dirichlet (11) and "Neumann" (12) problems, on the perforated domain $\Omega_{\rho}$, by

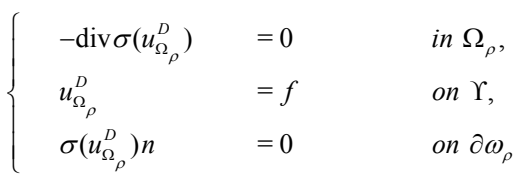

and

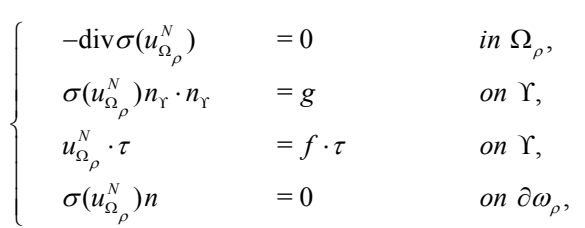

Where $n$ denotes the outward normal to $\Omega_{\rho}$ on the boundary $\partial \omega_{\rho}$. It should be reminded that for $\rho=0$, we have $\omega \rho=\varnothing$ and $\Omega_{0}=\Omega$.

One can remark that $\omega_{\rho}$ coincides with the actual cavity $\mathbb{O}(1)$ where there is no misfit between both Dirichlet (11) and "Neumann" (12) problems, that is when $u_{\Omega}^{D}=u_{\Omega}^{N}$ in $\Omega$. According to this observation, we propose to recover $\mathbb{O}$ by minimizing an energy gap cost functional, namely the following misfit Kohn-Vogelius functional (4) defined by

$$
j\left(\Omega_{\rho}\right)=\mathcal{J}\left(u_{\Omega_{\rho}}^{D}, u_{\Omega_{\rho}}^{N}\right)=\frac{1}{2} \int_{\Omega_{\rho}}\left(\sigma\left(u_{\Omega_{\rho}}^{D}\right)-\sigma\left(u_{\Omega_{\rho}}^{N}\right)\right):\left(\varepsilon\left(u_{\Omega_{\rho}}^{D}\right)-\varepsilon\left(u_{\Omega_{\rho}}^{N}\right)\right) d x,
$$

in the presence of a single hole $\omega_{\rho} \subset \Omega$. Above, for both Dirichlet
(11) and "Neumann" (12) problems, we impose a homogeneous Neumann boundary condition on $\partial \omega_{\rho}$ which means that $\omega_{\rho}$ represents a perforation, namely a cavity in mathematical concept.

The so-called Kohn-Vogelius criterion [19] (13) has already been investigated in the topological gradient context for the detection of cracks [20] in the steady-state heat equation, for the localization of small cavities in Stokes flow [12] and for the shape reconstruction of inclusions in an inverse conductivity problem [21].

The computation of the topological gradient exposed below is inspired by the paper [10] and let us point out that the main contribution in this paper relies not only on the detection of cavities from partially overdetermined boundary data but also on the use of the error functional (13), also known as an energetic least-squares functional $[3,4,8]$. Indeed, the aim here is to derive an asymptotic expansion for the cost functional $j$ (13) following the same procedure outlined in the previous section, that is, to study the variation of the design functional $\mathbb{I}$ with respect to the creation of small hole. However, the tools presented in the previous section cannot be applied directly as a fixed functional space $\mathbb{V}$ is required. Indeed, the displacements $u_{\Omega}^{D}$ (11) and $u_{\Omega_{\rho}}^{N}$ (12) are defined on the variable domain $\Omega_{\rho}$ dependant on $\rho$.

Let us mention that this question was solved in the context of shape gradient method by the means of a fixed domain [7], the so-called reference domain $\Omega$ and a bi-Lipshitz map between this reference domain $\Omega$ and the perturbed one.

Nevertheless, a domain truncation technique [10,14] comes to help in the topological gradient context which allows us to construct a functional space independent of $\rho$. Hence, the topological gradient method can be viewed as an extension of the classical shape gradient method $[3,4,7,8,22]$ with a difficulty concerning the lack of homeomorphism map between the safe domain and the perforated one $\Omega_{\rho}$ since the domains $\Omega$ and $\Omega_{\rho}$ haven't the same topology.

\section{Domain truncation technique}

Let $R>0$ be such that $\overline{B\left(x_{0}, R\right)} \subset \Omega$ and $\omega_{\rho} \subset B\left(x_{0}, R\right)$. Then, the truncated open set $\Omega_{R}$ (Figure 2) is defined by

$$
\Omega_{R}=\Omega \backslash \overline{B\left(x_{0}, R\right)} .
$$

Let us denote by $D_{\rho}$ the open set $B\left(x_{0}, R\right) \backslash \overline{\omega_{\rho}}$ (Figure 2).

In order to provide an asymptotic expansion of the functional $j$ (13), we need, first of all, an asymptotic expansion of both problems (11) and (12).

The Dirichlet problem: For $\rho \geq 0$, let us define the Dirichlet-toNeumann operator $T_{\rho}$, needed in the sequel, by
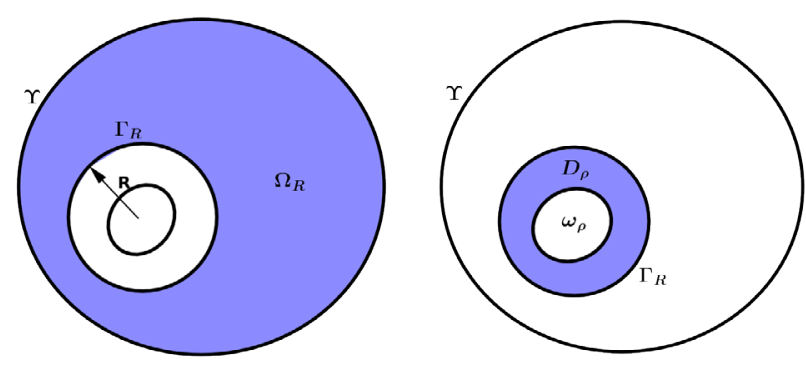

Figure 2: The truncated domain. 


$$
\begin{aligned}
T_{\rho}:\left[H^{\frac{1}{2}}\left(\Gamma_{R}\right)\right]^{2} & \rightarrow\left[H^{-\frac{1}{2}}\left(\Gamma_{R}\right)\right]^{2} \\
\varphi & \mapsto T_{\rho} \varphi=\sigma\left(u_{\rho}^{\varphi}\right) n,
\end{aligned}
$$

where $u_{\rho}^{\varphi}$ is the solution to the problem

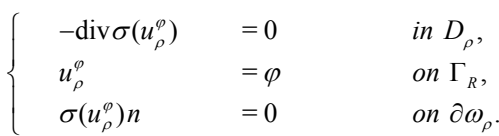

Above, $\Gamma_{R}$ is the boundary of $B\left(x_{0}, R\right)$. The normal $n$ is chosen outward to $D$ on $\partial \omega_{\rho}$ and $\Gamma_{R}$, regardless of whether $D_{\rho}$ or $\Omega_{R}$ are considered.

Hence, the displacement $u_{\rho}^{D}$ is defined for $\rho \geq 0$ as the solution of the truncated problem: Find $u_{\rho}^{D}$ such that

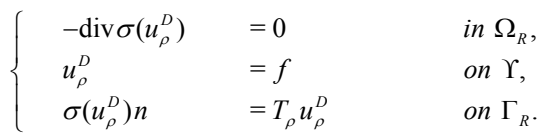

The problem (14) can be stated in its variational formulation as following:

$$
\begin{aligned}
& \text { Find } u_{\rho}^{D} \in\left[H^{1}\left(\Omega_{R}\right)\right]^{2} ; u_{\rho}^{D}=f \text { on } \Upsilon \text { such that } \\
& a_{\rho}^{D}\left(u_{\rho}^{D}, v\right)=l^{D}(v) \quad \forall v \in \mathcal{V}_{R}^{D},
\end{aligned}
$$

where the functional space $a_{\rho}^{D}$ and the bilinear form $a_{\rho}^{D}$ are defined by

$$
\begin{aligned}
& \mathcal{V}_{R}^{D}=\left\{u \in\left[H^{1}\left(\Omega_{R}\right)\right]^{2} ; \quad u=0 \text { on } \Upsilon\right\} \\
& \text { and } \quad a_{\rho}^{D}(u, v)=\int_{\Omega_{R}} \sigma(u): \varepsilon(v) d x+\int_{\Gamma_{R}} T_{\rho} u \cdot v d \gamma(x) .
\end{aligned}
$$

Here, $l^{\mathrm{D}} 0$.

The reader is referred to [10] to prove the symmetry, continuity and coercivity of $a_{\rho}^{D}$. Let us remind a standard result in PDE theory.

Proposition 1: Problems (11) and (14) have a unique solution. Moreover, the solution $u_{\rho}^{D}$ to problem (14) is the restriction to $\Omega_{R}$ of the solution $u_{\Omega_{\rho}}^{D}$ to problem (11) [13].

Proof: One can follow the same lines as in the Helmholtz problem [14].

The "Neumann" problem: Let $\Omega_{R}, \Gamma_{R}, D_{\rho}$ and the operator $T_{\rho}$ be as previously defined. Then, the displacement $u_{\rho}^{N}$ is defined for $\rho \geq 0$ as the solution of the truncated problem: Find $u_{\rho}^{N}$ such that

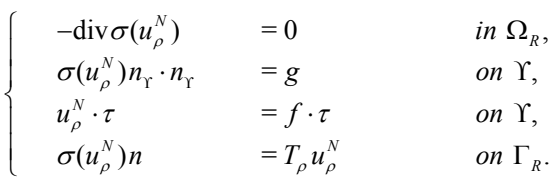

The variational formulation associated to problem (17) is the following:

Find $u_{\rho}^{N} \in\left[H^{1}\left(\Omega_{R}\right)\right]^{2} ; u_{\rho}^{N} \cdot \tau=f \cdot \tau$ on $\Upsilon$ such that

$$
a_{\rho}^{N}\left(u_{\rho}^{N}, v\right)=l^{N}(v) \quad \forall v \in \mathcal{V}_{R}^{N}
$$

where the functional space $\mathcal{V}_{R}^{N}$, the bilinear form $a_{\rho}^{N}$ and the linear form $l^{N}$ are defined by

$$
\begin{aligned}
& \mathcal{V}_{R}^{N}=\left\{u \in\left[H^{1}\left(\Omega_{R}\right)\right]^{2} ; u \cdot \tau=0 \text { on } \Upsilon\right\}, \\
& a_{\rho}^{N}(u, v)=\int_{\Omega_{R}} \sigma(u): \varepsilon(v) d x+\int_{\Gamma_{R}} T_{\rho} u \cdot v d \gamma(x)
\end{aligned}
$$

$$
\text { and } l^{N}(v)=\int_{\mathrm{r}} g\left(v \cdot n_{\mathrm{r}}\right) \text {. }
$$

Since $a_{\rho}^{N} \equiv a_{\rho}^{D}, a_{\rho}^{N}$ is symmetric, continuous and coercive and one can easily prove the continuity of the linear form $l^{N}$.

Then, we have the same standard result as the one related to the Dirichlet problem (Proposition 1), that is:

Proposition 2: Problems (12) and (17) have a unique solution. Moreover, the solution $u_{\rho}^{N}$ to problem (17) is the restriction to $\Omega_{R}$ of the solution $u_{\Omega_{\rho}}^{N}$ to problem (12).

The fixed Hilbert space $\left[H^{1}\left(\Omega_{R}\right)\right]^{2}$ required by the adjoint method previously presented in the second section is now available. For $u_{\rho}^{D} \in\left[H^{1}\left(\Omega_{R}\right)\right]^{2}$, let $\widetilde{u_{\rho}^{D}} \in\left[H^{1}\left(\Omega_{\rho}\right)\right]^{2}$ be the extension of $u_{\rho}^{D}$ which coincides with $u_{\rho}^{D}$ on $\Omega_{R}$ and $\Gamma_{R}$ and which satisfies

$$
\left\{\begin{array}{lll}
-\operatorname{div} \sigma\left(\widetilde{u_{\rho}^{D}}\right) & =0 & \text { in } D_{\rho}, \\
\sigma\left(\widetilde{u_{\rho}^{D}}\right) n & =0 & \text { on } \partial \omega_{\rho} .
\end{array}\right.
$$

In the same way, for $u_{\rho}^{N} \in\left[H^{1}\left(\Omega_{R}\right)\right]^{2}$, let $\widetilde{u_{\rho}^{N}} \in\left[H^{1}\left(\Omega_{\rho}\right)\right]^{2}$ be the extension of $u_{\rho}^{N}$ which coincides with $u_{\rho}^{N}$ on $\Omega_{R}$ and $\Gamma_{R}$ and which satisfies

$$
\left\{\begin{array}{lll}
-\operatorname{div} \sigma\left(\widetilde{u_{\rho}^{N}}\right) & =0 & \text { in } D_{\rho}, \\
\sigma\left(\widetilde{u_{\rho}^{N}}\right) n & =0 & \text { on } \partial \omega_{\rho} .
\end{array}\right.
$$

Then, a function $\mathcal{J}_{\rho}$ can be defined on $\left[H^{1}\left(\Omega_{R}\right)\right]^{2} \times\left[H^{1}\left(\Omega_{R}\right)\right]^{2}$ by $\mathcal{J}_{\rho}\left(u_{\rho}^{D}, u_{\rho}^{N}\right)=\mathcal{J}\left(\widetilde{u_{\rho}^{D}}, \widetilde{u_{\rho}^{N}}\right)$

and from the previous proposition 1 and 2, it follows that

$j\left(\Omega_{\rho}\right)=\mathcal{J}\left(u_{\Omega_{\rho}}^{D}, u_{\Omega_{\rho}}^{N}\right)=\mathcal{J}_{\rho}\left(u_{\rho}^{D}, u_{\rho}^{N}\right)$.

\section{The main result}

We are now able to prove the main result of our work available in the case of spherical hole.

Theorem 2: The function $j$ has the following asymptotic expansion

$$
j\left(\Omega_{\rho}\right)=j(\Omega)+\rho^{2}\left[\delta_{a^{D}}\left(u_{0}^{D}, v_{0}^{D}\right)+\delta_{a^{N}}\left(u_{0}^{N}, v_{0}^{N}\right)+\delta_{\mathcal{J}}\left(u_{0}^{D}, u_{0}^{N}\right)\right]+o\left(\rho^{2}\right),
$$

where

$$
\begin{aligned}
& \delta_{a^{D}}\left(u_{0}^{D}, v_{0}^{D}\right)=-\frac{\pi(\mu+\eta)}{2 \eta \mu}\left[4 \mu \sigma\left(u_{0}^{D}\right): \varepsilon\left(v_{0}^{D}\right)+(\eta-2 \mu) \operatorname{tr} \sigma\left(u_{0}^{D}\right) \operatorname{tr} \varepsilon\left(v_{0}^{D}\right)\right], \\
& \delta_{a^{N}}\left(u_{0}^{N}, v_{0}^{N}\right)=-\frac{\pi(\mu+\eta)}{2 \eta \mu}\left[4 \mu \sigma\left(u_{0}^{N}\right): \varepsilon\left(v_{0}^{N}\right)+(\eta-2 \mu) \operatorname{tr} \sigma\left(u_{0}^{N}\right) \operatorname{tr} \varepsilon\left(v_{0}^{N}\right)\right]
\end{aligned}
$$

and

$$
\delta_{\mathcal{J}}\left(u_{0}^{D}, u_{0}^{N}\right)=-\frac{\pi}{2}\left[\sigma\left(u_{0}^{D}\right)\left(x_{0}\right): \varepsilon\left(u_{0}^{D}\right)\left(x_{0}\right)+\sigma\left(u_{0}^{N}\right)\left(x_{0}\right): \varepsilon\left(u_{0}^{N}\right)\left(x_{0}\right)\right] .
$$

Above,

$\eta=\lambda+\mu$ (plane strain $)$

$v_{0}^{D} \in \mathcal{V}_{0}^{D}$ is the solution to the adjoint equation associated to the Dirichlet problem (9)

$$
a_{0}^{D}\left(w, v_{0}^{D}\right)=-\partial_{{ }_{u}^{D}} \mathcal{J}\left(u_{0}^{D}, u_{0}^{N}\right) w \quad \forall w \in \mathcal{V}_{0}^{D},
$$

$v_{0}^{N} \in \mathcal{V}_{0}^{N}$ is the solution to the adjoint equation associated to the Neumann problem (10)

$$
a_{0}^{N}\left(w, v_{0}^{N}\right)=-\partial_{u_{0}^{N}} \mathcal{J}\left(u_{0}^{D}, u_{0}^{N}\right) w \quad \forall w \in \mathcal{V}_{0}^{N} .
$$

1) Variation of the bilinear form $a_{\rho}^{D}$ (Dirichlet problem)

The variation of the bilinear form $a_{\rho}^{D}$ (16) defined by 
$a_{\rho}^{D}(u, v)=\int_{\Omega_{R}} \sigma(u): \varepsilon(v) d x+\int_{\Gamma_{R}} T_{\rho} u \cdot v d \gamma(x)$

reads

$a_{\rho}^{D}(u, v)-a_{0}^{D}(u, v)=\int_{\Gamma_{R}}\left(T_{\rho}-T_{0}\right) u \cdot v d \gamma(x)$.

As a consequence, one has only to examine $\left(T_{\rho}-T_{0}\right) \phi$ for $\varphi \in\left[H^{\frac{1}{2}}\left(\Gamma_{R}\right)\right]^{2}$. Indeed, one would like to find an operator $\delta_{T} \in \mathcal{L}\left(H^{\frac{1}{2}}\left(\Gamma_{R}\right)^{2} ; H^{-\frac{1}{2}}\left(\Gamma_{R}\right)^{2}\right)$ such that

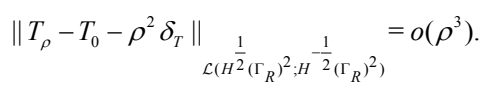

Hence, we define $\delta_{a^{D}}$ by

$$
\delta_{a} D(u, v)=\int_{\Gamma_{R}} \delta_{T} u \cdot v d \gamma(x) \quad \forall u, v \in\left[H^{1}\left(\Omega_{R}\right)\right]^{2}
$$

and we get that

$$
\left\|a_{\rho}^{D}-a_{0}^{D}-\rho^{2} \delta_{a^{D}}\right\|_{\mathcal{L}_{2}\left[H^{1}\left(\Omega_{R}\right]^{2}\right)}=o\left(\rho^{3}\right)
$$

Making use of the same procedure outlined in [10], one can get the expression (22).

\section{2) Variation of the bilinear form $a_{\rho}^{N}$ (Neumann problem)}

We remark that the same bilinear form $a$ is available for the Dirichlet and the Neumann problem, namely $a_{\rho}^{D} \equiv a_{\rho}^{N}$. As a consequence, the same variation (23) of $a_{\rho}^{N}$ follows.

\section{3) Variation of the linear form $l^{\mathrm{D}}$ and $l^{\mathrm{N}}$}

Since $l^{N}$ is independent of $\rho$, it follows trivially that $\delta_{l^{N}} \equiv 0$ and since $l^{D} \equiv 0$, then $\delta_{l D} \equiv 0$.

\section{4) Variation of the cost functional}

Let us now focus our attention on the variation of the KohnVogelius functional given by

$$
\mathcal{J}\left(u_{\Omega_{\rho}}^{D}, u_{\Omega_{\rho}}^{N}\right)=\frac{1}{2} \int_{\Omega_{\rho}}\left(\sigma\left(u_{\Omega_{\rho}}^{D}\right)-\sigma\left(u_{\Omega_{\rho}}^{N}\right)\right):\left(\varepsilon\left(u_{\Omega_{\rho}}^{D}\right)-\varepsilon\left(u_{\Omega_{\rho}}^{N}\right)\right) d x .
$$

This functional $\mathcal{J}$ can be decomposed as

$$
\begin{aligned}
& \mathcal{J}\left(u_{\Omega_{\rho}}^{D}, u_{\Omega_{\rho}}^{N}\right)=\mathcal{J}_{D}\left(u_{\Omega_{\rho}}^{D}\right)+\mathcal{J}_{N}\left(u_{\Omega_{\rho}}^{N}\right)+\mathcal{J}_{D N}\left(u_{\Omega_{\rho}}^{D}, u_{\Omega_{\rho}}^{N}\right), \\
& \text { where } \begin{cases}\mathcal{J}_{D}\left(u_{\Omega_{\rho}}^{D}\right) & =\frac{1}{2} \int_{\Omega_{\rho}} \sigma\left(u_{\Omega_{\rho}}^{D}\right): \varepsilon\left(u_{\Omega_{\rho}}^{D}\right) d x, \\
\mathcal{J}_{N}\left(u_{\Omega_{\rho}}^{N}\right) & =\frac{1}{2} \int_{\Omega_{\rho}} \sigma\left(u_{\Omega_{\rho}}^{N}\right): \varepsilon\left(u_{\Omega_{\rho}}^{N}\right) d x, \\
\mathcal{J}_{D N}\left(u_{\Omega_{\rho}}^{D}, u_{\Omega_{\rho}}^{N}\right) & =-\int_{\Omega_{\rho}} \sigma\left(u_{\Omega_{\rho}}^{D}\right): \varepsilon\left(u_{\Omega_{\rho}}^{N}\right) d x,\end{cases}
\end{aligned}
$$

Variation of $\mathcal{J}_{D}$ : The variation of $\mathcal{J}_{D}$ reads

$$
\begin{aligned}
& \mathcal{J}_{D}\left(u_{\Omega_{\rho}}^{D}\right)-\mathcal{J}_{D}\left(u_{0}^{D}\right)=\frac{1}{2} \int_{\Omega_{\rho}} \sigma\left(u_{\Omega_{\rho}}^{D}\right): \varepsilon\left(u_{\Omega_{\rho}}^{D}\right) d x-\frac{1}{2} \int_{\Omega} \sigma\left(u_{0}^{D}\right): \varepsilon\left(u_{0}^{D}\right) d x, \\
& =\frac{1}{2} \int_{\Omega_{\rho}} \sigma\left(u_{\Omega_{\rho}}^{D}-u_{0}^{D}\right): \varepsilon\left(u_{\Omega_{\rho}}^{D}\right) d x \\
& +\frac{1}{2} \int_{\Omega_{\rho}} \sigma\left(u_{\Omega_{\rho}}^{D}-u_{0}^{D}\right): \varepsilon\left(u_{0}^{D}\right) d x-\frac{1}{2} \int_{\omega_{\rho}} \sigma\left(u_{0}^{D}\right): \varepsilon\left(u_{0}^{D}\right) d x .
\end{aligned}
$$

Using the Green formula, one can get from (11) that

$\frac{1}{2} \int_{\Omega_{\rho}} \sigma\left(u_{\Omega_{\rho}}^{D}-u_{0}^{D}\right): \varepsilon\left(u_{\Omega_{\rho}}^{D}\right) d x=0$.

Then, it follows that

$$
\mathcal{J}_{D}\left(u_{\Omega_{\rho}}^{D}\right)-\mathcal{J}_{D}\left(u_{0}^{D}\right)=\frac{1}{2} \int_{\Omega_{\rho}} \sigma\left(u_{\Omega_{\rho}}^{D}-u_{0}^{D}\right): \varepsilon\left(u_{0}^{D}\right) d x-\frac{1}{2} \int_{\omega_{\rho}} \sigma\left(u_{0}^{D}\right): \varepsilon\left(u_{0}^{D}\right) d x .
$$

Variation of $\mathcal{J}_{N}$ : The variation of $\mathcal{J}_{N}$ reads

$\mathcal{J}_{N}\left(u_{\Omega_{\rho}}^{N}\right)-\mathcal{J}_{N}\left(u_{0}^{N}\right)=\frac{1}{2} \int_{\Omega_{\rho}} \sigma\left(u_{\Omega_{\rho}}^{N}\right): \varepsilon\left(u_{\Omega_{\rho}}^{N}\right) d x-\frac{1}{2} \int_{\Omega} \sigma\left(u_{0}^{N}\right): \varepsilon\left(u_{0}^{N}\right) d x$,

$=\frac{1}{2} \int_{\Omega_{\rho}} \sigma\left(u_{\Omega_{\rho}}^{N}-u_{0}^{N}\right): \varepsilon\left(u_{\Omega_{\rho}}^{N}\right) d x$

$+\frac{1}{2} \int_{\Omega_{\rho}} \sigma\left(u_{\Omega_{\rho}}^{N}-u_{0}^{N}\right): \varepsilon\left(u_{0}^{N}\right) d x-\frac{1}{2} \int_{\omega_{\rho}} \sigma\left(u_{0}^{N}\right): \varepsilon\left(u_{0}^{N}\right) d x$.

Using the Green formula applied to the problem (12), we obtain $\frac{1}{2} \int_{\Omega_{\rho}} \sigma\left(u_{\Omega_{\rho}}^{N}-u_{0}^{N}\right): \varepsilon\left(u_{\Omega_{\rho}}^{N}\right) d x=\frac{1}{2} \int_{\Upsilon} \sigma\left(u_{\Omega_{\rho}}^{N}\right) n_{\Upsilon} \cdot\left(u_{\Omega_{\rho}}^{N}-u_{0}^{N}\right) d s$.

Since $u_{\Omega_{\rho}}^{N} \cdot \tau=u_{0}^{N} \cdot \tau=f \cdot \tau$ and $\sigma\left(u_{\Omega_{\rho}}^{N}\right) n_{\Upsilon} \cdot n_{\Upsilon}=g$ on $\Upsilon$, we have $\frac{1}{2} \int_{\Upsilon} \sigma\left(u_{\Omega_{\rho}}^{N}\right) n_{\Upsilon} \cdot\left(u_{\Omega_{\rho}}^{N}-u_{0}^{N}\right) d s=\frac{1}{2} \int_{\Upsilon} g\left[\left(u_{\Omega_{\rho}}^{N}-u_{0}^{N}\right) \cdot n_{\Upsilon}\right] d s$.

Then, the following equality holds

$\frac{1}{2} \int_{\Omega_{\rho}} \sigma\left(u_{\Omega_{\rho}}^{N}-u_{0}^{N}\right): \varepsilon\left(u_{\Omega_{\rho}}^{N}\right) d x=\frac{1}{2} \int_{\mathrm{r}} g\left[\left(u_{\Omega_{\rho}}^{N}-u_{0}^{N}\right) \cdot n_{\mathrm{\Upsilon}}\right] d s$.

Hence, we get

$\mathcal{J}_{N}\left(u_{\Omega_{\rho}}^{N}\right)-\mathcal{J}_{N}\left(u_{0}^{N}\right)=\frac{1}{2} \int_{\Omega_{\rho}} \sigma\left(u_{\Omega_{\rho}}^{N}-u_{0}^{N}\right): \varepsilon\left(u_{0}^{N}\right) d x+\frac{1}{2} \int_{\Upsilon} g\left[\left(u_{\Omega_{\rho}}^{N}-u_{0}^{N}\right) \cdot n_{\Upsilon}\right] d s$

$-\frac{1}{2} \int_{\omega_{\rho}} \sigma\left(u_{0}^{N}\right): \varepsilon\left(u_{0}^{N}\right) d x$.

Variation of $\mathcal{J}_{D N}$ : The variation of $\mathcal{J}_{D N}$ reads

$\mathcal{J}_{D N}\left(u_{\Omega_{\rho}}^{D}, u_{\Omega_{\rho}}^{N}\right)-\mathcal{J}_{D N}\left(u_{0}^{D}, u_{0}^{N}\right)=-\int_{\Omega_{\rho}} \sigma\left(u_{\Omega_{\rho}}^{D}\right): \varepsilon\left(u_{\Omega_{\rho}}^{N}\right) d x+\int_{\Omega} \sigma\left(u_{0}^{D}\right): \varepsilon\left(u_{0}^{N}\right) d x$.

Using the Green formula applied, on the one hand, to the problem (12) we obtain

$-\int_{\Omega_{\rho}} \sigma\left(u_{\Omega_{\rho}}^{D}\right): \varepsilon\left(u_{\Omega_{\rho}}^{N}\right) d x=-\int_{\Upsilon} \sigma\left(u_{\Omega_{\rho}}^{N}\right) n_{\Upsilon} \cdot f d s$

and on the other hand, to the problem (10) we get

$\int_{\Omega} \sigma\left(u_{0}^{D}\right): \varepsilon\left(u_{0}^{N}\right) d x=\int_{\Upsilon} \sigma\left(u_{0}^{N}\right) n_{\Upsilon} \cdot f d s$.

Then, we have

$\mathcal{J}_{D N}\left(u_{\Omega_{\rho}}^{D}, u_{\Omega_{\rho}}^{N}\right)-\mathcal{J}_{D N}\left(u_{0}^{D}, u_{0}^{N}\right)=\int_{\Upsilon} \sigma\left(u_{0}^{N}-u_{\Omega_{\rho}}^{N}\right) n_{\Upsilon} \cdot f d s$.

Since $\sigma\left(u_{0}^{N}\right) n_{\Upsilon} \cdot n_{\Upsilon}=\sigma\left(u_{\Omega_{\rho}}^{N}\right) n_{\Upsilon} \cdot n_{\Upsilon}=g$ on $\Upsilon$, it follows that

$\mathcal{J}_{D N}\left(u_{\Omega_{\rho}}^{D}, u_{\Omega_{\rho}}^{N}\right)-\mathcal{J}_{D N}\left(u_{0}^{D}, u_{0}^{N}\right)=\int_{\Upsilon}\left[\sigma\left(u_{0}^{N}-u_{\Omega_{\rho}}^{N}\right) n_{\Upsilon} \cdot \tau\right](f \cdot \tau) d s$.

Combining the variations (27), (28) and (29), the variation of the functional $\mathcal{J}$ becomes

$\mathcal{J}\left(u_{\Omega_{\rho}}^{D}, u_{\Omega_{\rho}}^{N}\right)-\mathcal{J}\left(u_{0}^{D}, u_{0}^{N}\right)$

$=\mathcal{J}_{D}\left(u_{\Omega_{\rho}}^{D}\right)-\mathcal{J}_{D}\left(u_{0}^{D}\right)+\mathcal{J}_{D N}\left(u_{\Omega_{\rho}}^{D}, u_{\Omega_{\rho}}^{N}\right)-\mathcal{J}_{D N}\left(u_{0}^{D}, u_{0}^{N}\right)+\mathcal{J}_{N}\left(u_{\Omega_{\rho}}^{N}\right)-\mathcal{J}_{N}\left(u_{0}^{N}\right)$,

$=\frac{1}{2} \int_{\Omega_{\rho}} \sigma\left(u_{\Omega_{\rho}}^{D}-u_{0}^{D}\right): \varepsilon\left(u_{0}^{D}\right) d x-\frac{1}{2} \int_{\omega_{\rho}} \sigma\left(u_{0}^{D}\right): \varepsilon\left(u_{0}^{D}\right) d x$

$+\int_{\Upsilon}\left[\sigma\left(u_{0}^{N}-u_{\Omega_{\rho}}^{N}\right) n_{\mathrm{\Upsilon}} \cdot \tau\right](f \cdot \tau) d s+\frac{1}{2} \int_{\Omega_{\rho}} \sigma\left(u_{\Omega_{\rho}}^{N}-u_{0}^{N}\right): \varepsilon\left(u_{0}^{N}\right) d x$

$+\frac{1}{2} \int_{\Upsilon} g\left[\left(u_{\Omega_{\rho}}^{N}-u_{0}^{N}\right) \cdot n_{\Upsilon}\right] d s-\frac{1}{2} \int_{\omega_{\rho}} \sigma\left(u_{0}^{N}\right): \varepsilon\left(u_{0}^{N}\right) d x$,

$=D \mathcal{J}\left(u_{0}^{D}, u_{0}^{N}\right)\left(u_{\Omega_{\rho}}^{D}-u_{0}^{D}, u_{\Omega_{\rho}}^{N}-u_{0}^{N}\right)-\frac{1}{2} \int_{\omega_{\rho}} \sigma\left(u_{0}^{D}\right): \varepsilon\left(u_{0}^{D}\right) d x-\frac{1}{2} \int_{\omega_{\rho}} \sigma\left(u_{0}^{N}\right): \varepsilon\left(u_{0}^{N}\right) d x$, where

$$
\begin{aligned}
& D \mathcal{J}\left(u_{0}^{D}, u_{0}^{N}\right)\left(u_{\Omega_{\rho}}^{D}-u_{0}^{D}, u_{\Omega_{\rho}}^{N}-u_{0}^{N}\right) \\
& =\frac{1}{2} \int_{\Omega_{\rho}} \sigma\left(u_{\Omega_{\rho}}^{D}-u_{0}^{D}\right): \varepsilon\left(u_{0}^{D}\right) d x+\frac{1}{2} \int_{\Omega_{\rho}} \sigma\left(u_{\Omega_{\rho}}^{N}-u_{0}^{N}\right): \varepsilon\left(u_{0}^{N}\right) d x
\end{aligned}
$$




$$
-\int_{\Upsilon}\left[\sigma\left(u_{\Omega_{\rho}}^{N}-u_{0}^{N}\right) n_{\Upsilon} \cdot \tau\right](f \cdot \tau) d s+\frac{1}{2} \int_{\Upsilon} g\left[\left(u_{\Omega_{\rho}}^{N}-u_{0}^{N}\right) \cdot n_{\Upsilon}\right] d s .
$$

Using the change of variables $x=x_{0}+\rho y$ with $x_{0}=0$, one can write

$$
\int_{\omega_{\rho}} \sigma\left(u_{0}^{D}\right): \varepsilon\left(u_{0}^{D}\right) d x=\rho^{2} \int_{\omega} \sigma\left(u_{0}^{D}\right)(\rho y): \varepsilon\left(u_{0}^{D}\right)(\rho y) d y,
$$$$
=\rho^{2} \int_{\omega} \sigma\left(u_{0}^{D}\right)(0): \varepsilon\left(u_{0}^{D}\right)(0) d y
$$$$
+2 \rho^{2} \int_{\omega}\left[\sigma\left(u_{0}^{D}\right)(\rho y)-\sigma\left(u_{0}^{D}\right)(0)\right]: \varepsilon\left(u_{0}^{D}\right)(0) d y
$$$$
+\rho^{2} \int_{\omega}\left[\sigma\left(u_{0}^{D}\right)(\rho y)-\sigma\left(u_{0}^{D}\right)(0)\right]:\left[\varepsilon\left(u_{0}^{D}\right)(\rho y)-\varepsilon\left(u_{0}^{D}\right)(0)\right] d y,
$$$$
=\rho^{2} \int_{\omega} \sigma\left(u_{0}^{D}\right)(0): \varepsilon\left(u_{0}^{D}\right)(0) d y+E_{1}(\rho)+E_{2}(\rho),
$$

where

$$
\begin{aligned}
& E_{1}(\rho)=2 \rho^{2} \int_{\omega}\left[\sigma\left(u_{0}^{D}\right)(\rho y)-\sigma\left(u_{0}^{D}\right)(0)\right]: \varepsilon\left(u_{0}^{D}\right)(0) d y \\
& \text { and } \quad E_{2}(\rho)=\rho \int\left[\sigma\left(u_{0}^{D}\right)(\rho y)-\sigma\left(u_{0}^{D}\right)(0)\right]:\left[\varepsilon\left(u_{0}^{D}\right)(\rho y)-\varepsilon\left(u_{0}^{D}\right)(0)\right] d y .
\end{aligned}
$$

By the Taylor expansion, using the fact that is regular in a neighborhood of 0 , namely $u_{0}^{D} \in C^{2}\left(\omega_{\rho}\right)$, one can prove that

$$
\int_{\omega}\left[\sigma\left(u_{0}^{D}\right)(\rho y)-\sigma\left(u_{0}^{D}\right)(0)\right]: \varepsilon\left(u_{0}^{D}\right)(0) d y=O(\rho)
$$

and so we obtain $E_{1}(\rho)=\mathrm{o}\left({ }^{2}\right)$.

In the same way, using the Taylor expansion and the regularity of $u_{0}^{D}$ in a neighborhood of the origin, we get that

$$
\int_{\omega}\left[\sigma\left(u_{0}^{D}\right)(\rho y)-\sigma\left(u_{0}^{D}\right)(0)\right]:\left[\varepsilon\left(u_{0}^{D}\right)(\rho y)-\varepsilon\left(u_{0}^{D}\right)(0)\right] d y=O\left(\rho^{2}\right)
$$

and so $E_{2}(\rho)=\mathrm{o}\left({ }^{2}\right)$. Then, it follows that

$$
\frac{1}{2} \int_{\omega_{\rho}} \sigma\left(u_{0}^{D}\right): \varepsilon\left(u_{0}^{D}\right) d x=\frac{1}{2} \rho^{2} \int_{\omega} \sigma\left(u_{0}^{D}\right)(0): \varepsilon\left(u_{0}^{D}\right)(0) d x+o\left(\rho^{2}\right) \text {. }
$$

Following the same lines outlined above, we derive

$$
\frac{1}{2} \int_{\omega_{\rho}} \sigma\left(u_{0}^{N}\right): \varepsilon\left(u_{0}^{N}\right) d x=\frac{1}{2} \rho^{2} \int_{\omega} \sigma\left(u_{0}^{N}\right)(0): \varepsilon\left(u_{0}^{N}\right)(0) d x+o\left(\rho^{2}\right) .
$$

Thus,

$$
\delta_{\mathcal{J}}\left(u_{0}^{D}, u_{0}^{N}\right)=-\frac{\pi}{2}\left[\sigma\left(u_{0}^{D}\right)\left(x_{0}\right): \varepsilon\left(u_{0}^{D}\right)\left(x_{0}\right)+\sigma\left(u_{0}^{N}\right)\left(x_{0}\right): \varepsilon\left(u_{0}^{N}\right)\left(x_{0}\right)\right] .
$$

All the assumptions of the adjoint method are satisfied and the topological asymptotic expansion is given by Theorem 1 .

\section{Numerical Experiments}

In spite of the lack of identifiability of the inverse problem under consideration(the identifiability was only proved in the case of monotonous cavities [7]), it is nevertheless useful to evaluate the efficiency of the proposed method through numerical experiments.

Therefore, this part aims to develop a one-shot reconstruction algorithm to numerically solve our cavities identification problem from partially overdetermined boundary data using the topological gradient method. In order to evaluate the topological gradient g, it is practically sufficient to solve the Dirichlet (9) and Neumann (10) state equations as well as the appropriate adjoint Dirichlet (25) and Neumann (26) state equations in the safe domain $\Omega$. Hence, an efficient and cheap topology optimization algorithm can be implemented in our case as one only needs to compute the solution of both Dirichlet and Neumann direct and adjoint problems. Since the function $j$ has to be minimized, one has to create holes at some points $x_{0}$ where the topological gradient $g\left(x_{0}\right)$, giving by

$$
g\left(x_{0}\right)=\delta_{a^{D}}\left(u_{0}^{D}, v_{0}^{D}\right)+\delta_{a^{N}}\left(u_{0}^{N}, v_{0}^{N}\right)+\delta_{\mathcal{J}}\left(u_{0}^{D}, u_{0}^{N}\right),
$$

is the most negative. In order to point out this feature, let us enumerate the steps of the topological optimization algorithm to be implemented.

\section{Algorithm}

Let $\Omega^{\text {true }}$ be a domain containing a cavity whose location and shape are to be retrieved from boundary measurements. The displacement $f$ and the normal component of the normal stress g are generated by a numerical computation of a direct problem over the domain $\Omega^{\text {true }}$ containing the cavity to recover (synthetic data).

Since the partially overdetermined boundary data $(f, g)$ are overspecified, the numerical procedure could be represented in the following algorithm stages.

1. Provide the initial domain $\Omega_{0}$ (the safe domain).

2. Solve the Dirichlet (9) and Neumann (10) direct problems in the safe domain $\Omega_{0}$.

3. Solve the Dirichlet (25) and Neumann (26) adjoint problems in the safe domain $\Omega_{0}$.

4. Compute the topological gradient $g(30)$.

5. Create holes at the points where the topological derivative is the most negative.

\section{Numerical tests}

In this subsection, we provide some numerical results related to the identification of cavities carried out with the algorithm described above.

We consider for all the following tests the same initial domain $\Omega_{0}$, namely the disc centered at the origin of radius $r_{0}=2$.

First case: In this case, the solution, namely the boundary of the cavity to recover, is the circle $C_{\text {exact }}$ centered at the origin with radius $R_{\text {exact }}=0.45$ and the partially overdetermined boundary data, that is the displacement field and the normal component of the normal stress are taken from the analytical expression $u_{\text {exact }}$ of the solution $u$ of the elasticity problem with complete data [3], given by

$$
u_{\text {exact }}(r)=\frac{C 1}{2} r+\frac{C 2}{r}
$$

where

$$
C 1=\frac{2(1+v)(1-2 v)}{E} A \text { and } C 2=\frac{1+v}{E} B
$$

Above, the constants $A$ and $B$ are determined by the boundary conditions, namely

$$
A=\frac{P_{1} r_{1}^{2}-P_{2} r_{0}^{2}}{r_{0}^{2}-r_{1}^{2}} \text { and } B=\frac{P_{1}-P_{2}}{r_{0}^{2}-r_{1}^{2}} r_{1}^{2} r_{0}^{2}
$$

where $r_{1}=R_{\text {exact }}, \sigma_{r}\left(r_{1}\right)=-P_{1}=0$ and $\sigma_{r}\left(r_{0}\right)=-P_{2}\left(\sigma_{r}\right.$ is the radial stress $)$.

Figure 3 shows the level lines of the topological gradient $g$ (30). We remark that the level set curve of $g$ related to the smallest value corresponds to the cavity to be recovered.

Second case: In the following cases, the measurements $f$ and $g$ are synthetic, i.e. generated by a numerical computation.

In this second case, we consider again a single cavity centered at (-1.6;-0.3) with radius $R_{\text {exact }}=0.3$. Figure 4 depicts the rough location of the cavity.

In contrast to the first and second cases focusing on the 

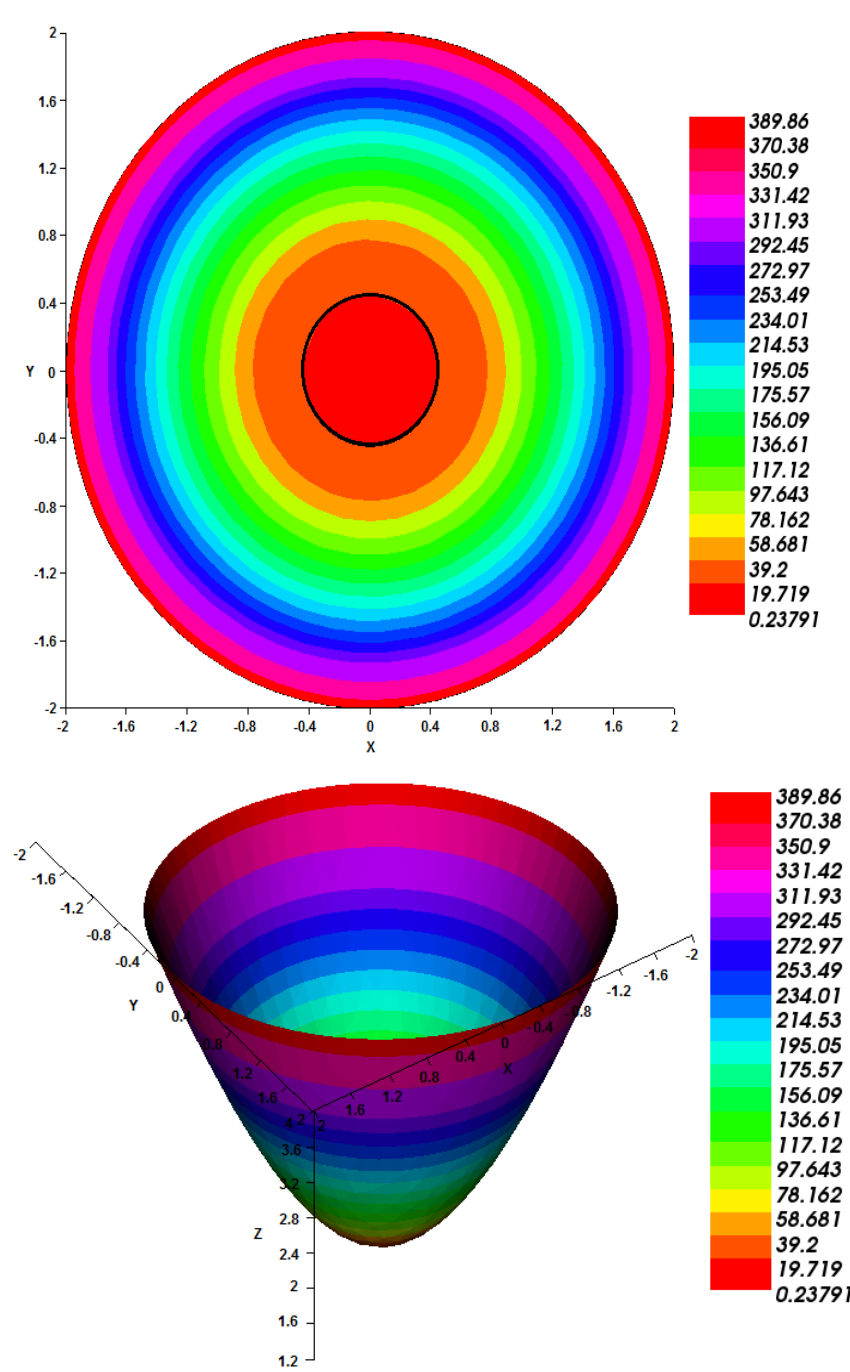

Figure 3: On the left: superposition of the actual cavity and the smallest isovalue of the topological gradient; on the right: the topological gradient $g$.

identification of a single cavity, the issue discussed in the next cases deals with multiple cavities detection insofar as the topological gradient does not depend on the number of cavities.

Third case: The aim of the third numerical experiment is to identify two cavities centered at $(-1.65 ; 0)$ and $(1.65 ; 0)$ with radius $R_{\text {exact }}=0.3$. From Figure 5, it turns out that an accurate detection is achieved.

Fourth case: For the numerical reconstruction in the last experiment, we focus on the case of a mechanical structure with four cavities, having the same radius $R_{\text {exact }}=0.3$ and centered at $(-1.65 ; 0)$, $(1.65 ; 0),(0 ;-1.65)$ and $(0 ; 1.65)$, to be recovered. The negative level lines of the topological gradient presented in Figure 6 coincide with the actual cavities.

Let us point that although the partially overdetermined boundary data lead additional difficulties especially in the numerical part, the detection of the cavities is efficient. Thus, these numerical illustrations demonstrate that the approach, namely the topological gradient method combined with the Kohn-Vogelius functional, proposed in this paper, is a reliable tool to recover cavities in mechanical structures.

\section{Conclusion}

Among several techniques proposed to solve the problem of detection of geometrical faults arising in elasticity framework, we resort, in this paper, to the topological gradient method which furnishes the sensitivity of a shape functional when modifying the topology of the domain with respect to the creation of a small hole.

We have proposed a one-shot approach based on the topological gradient method combined with the so-called Kohn-Vogelius gap cost functional. From the numerical results, the topological sensitivity method has been seen to be a powerful tool to be applied in topology optimization problems even in the case of partially overdetermined boundary data.

Moreover, the theoretical question related to the identifiability is still open since the uniqueness result was only derived for the case of monotonous cavities.

Although the process of the topological gradient method is not only cheap but also fast, it only allows to detect the rough location of faults. Hence, if one needs to determine a precise location, it should use some others approaches like level set method. A promising strategy [23] to
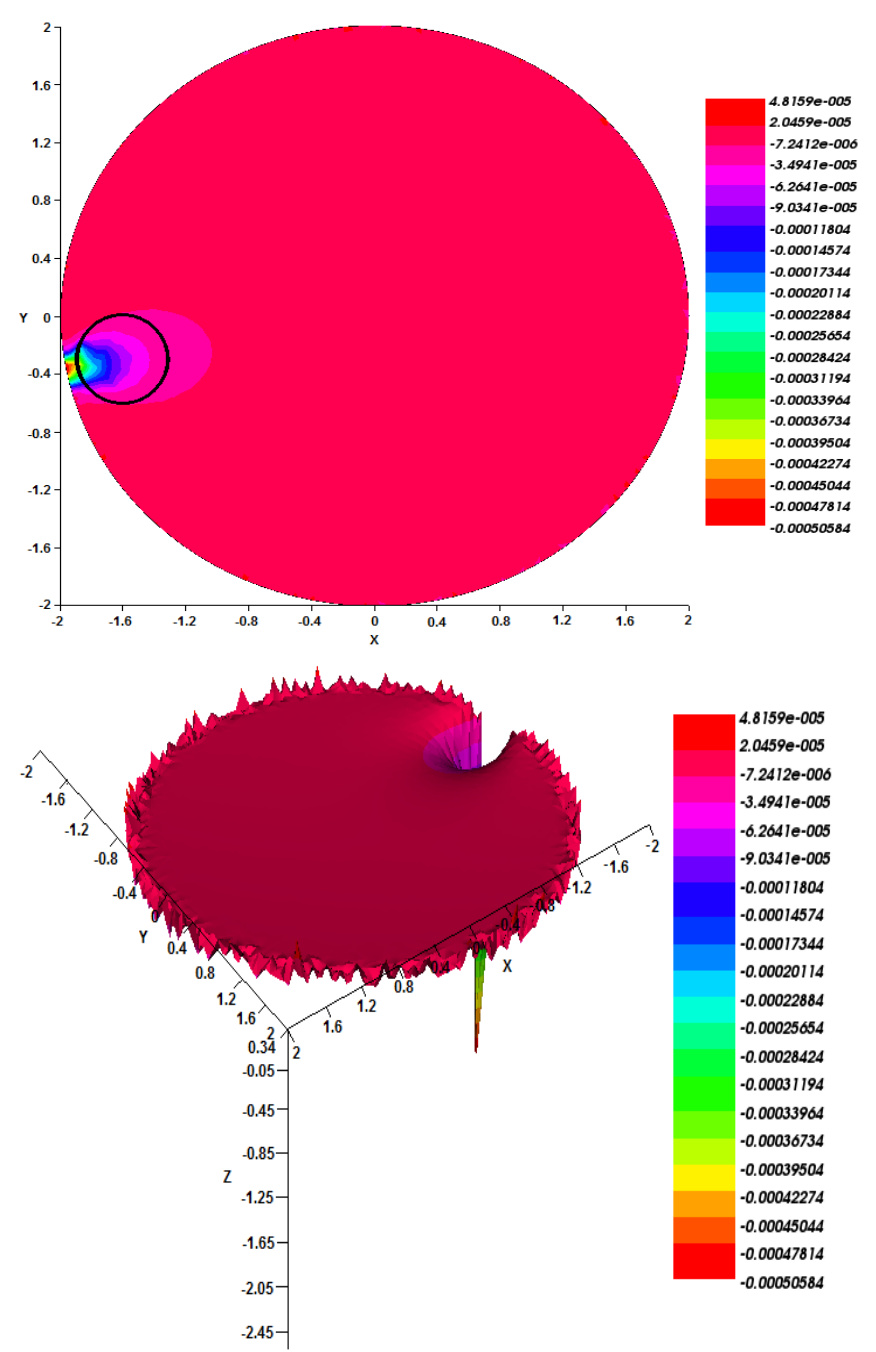

Figure 4: On the left: superposition of the actual cavity and negative level lines of the topological gradient; on the right: the topological gradient $g$. 
Citation: Jaiem E, Abda AB, Rjaibi B (2016) Cavities Identification from Partially Overdetermined Boundary Data in Linear Elasticity. J Appl Computat Math 5: 295. doi:10.4172/2168-9679.1000295
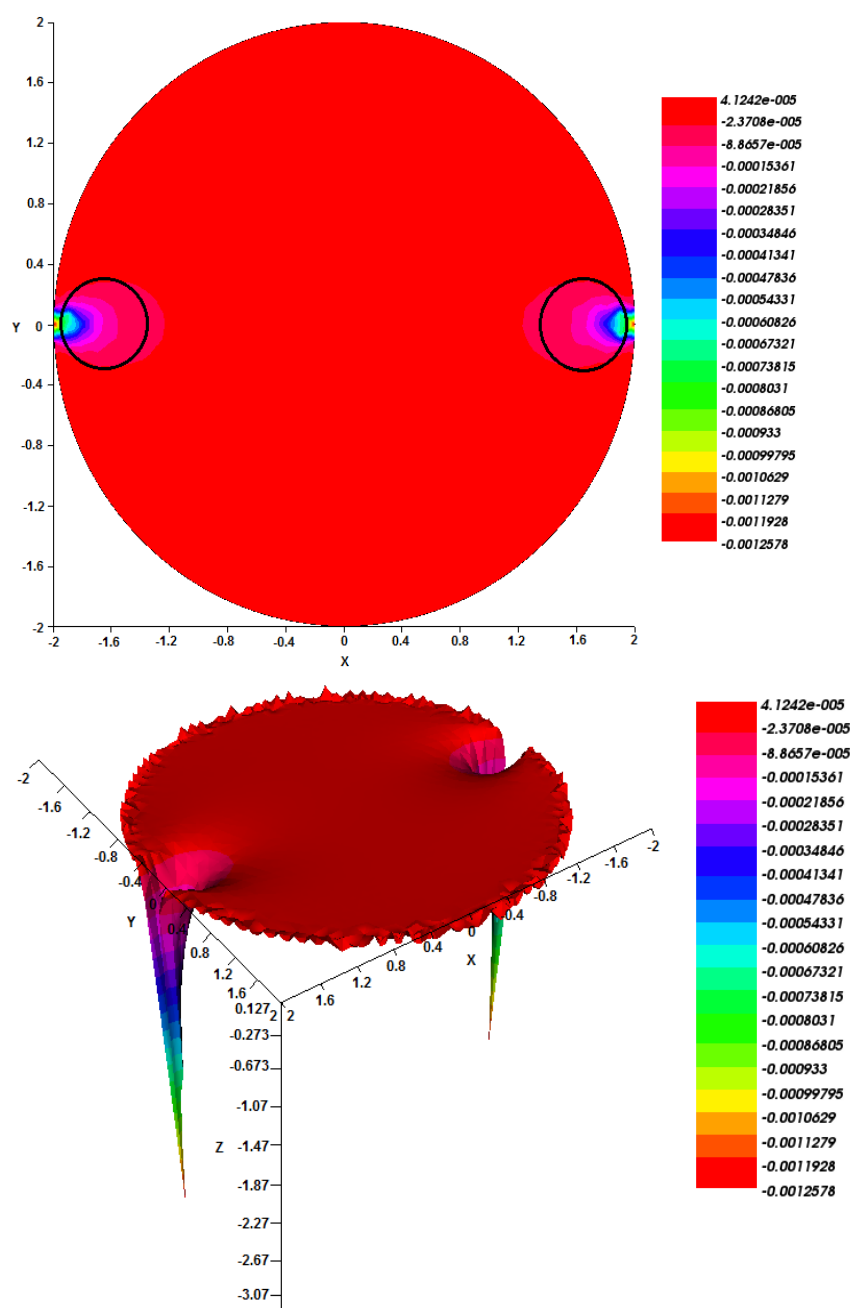

Figure 5: On the left: superposition of the actual cavities and negative leve lines of the topological gradient; on the right: the topological gradient $g$.

be explored is so to combine the level set method with the topological gradient one from which a good initial guess can be obtained.

\section{References}

1. Lemaitre J (1996) A course on damage mechanics. Springer Verlag Berlin Heidelberg.

2. Mishnaevsky JRL (2007) Computational Mesomechanics of Composites: Numerical analysis of the effect of microstructures of composite on their strength and damage resistance. John Wiley \& Sons, Ltd., England.

3. Ben Abda A, Jaïem E, Khalfallah S, Zine A (2015) An energy gap functional: Cavities identification in linear elasticity.

4. Jaïem E, Khalfallah S (2016) An energy-gap cost functional for cavities identification. Nonlinear Studies.

5. Ben Ameur H, Burger M, Hackl B (2007) Cavity identification in linear elasticity and thermoelasticity. Inverse Problems 30: 625-647.

6. Ben Ameur H, Burger M, Hackl B (2004) Level set methods for geometric inverse problems in linear elasticity. Inverse Problems 12: 673-696.

7. Jaïem E (2016) Shape derivative of an energy error functional: voids detection.

8. Ben Abda A, Bouchon F, Peichl GH, Sayeh M, Touzani R (2013) A DirichletNeumann cost functional approach for the Bernoulli problem. Journal of Engineering Mathematics 81: 157-176.

9. Schumacher A (1995) Topologieoptimierung von Bauteilstrukturen unter
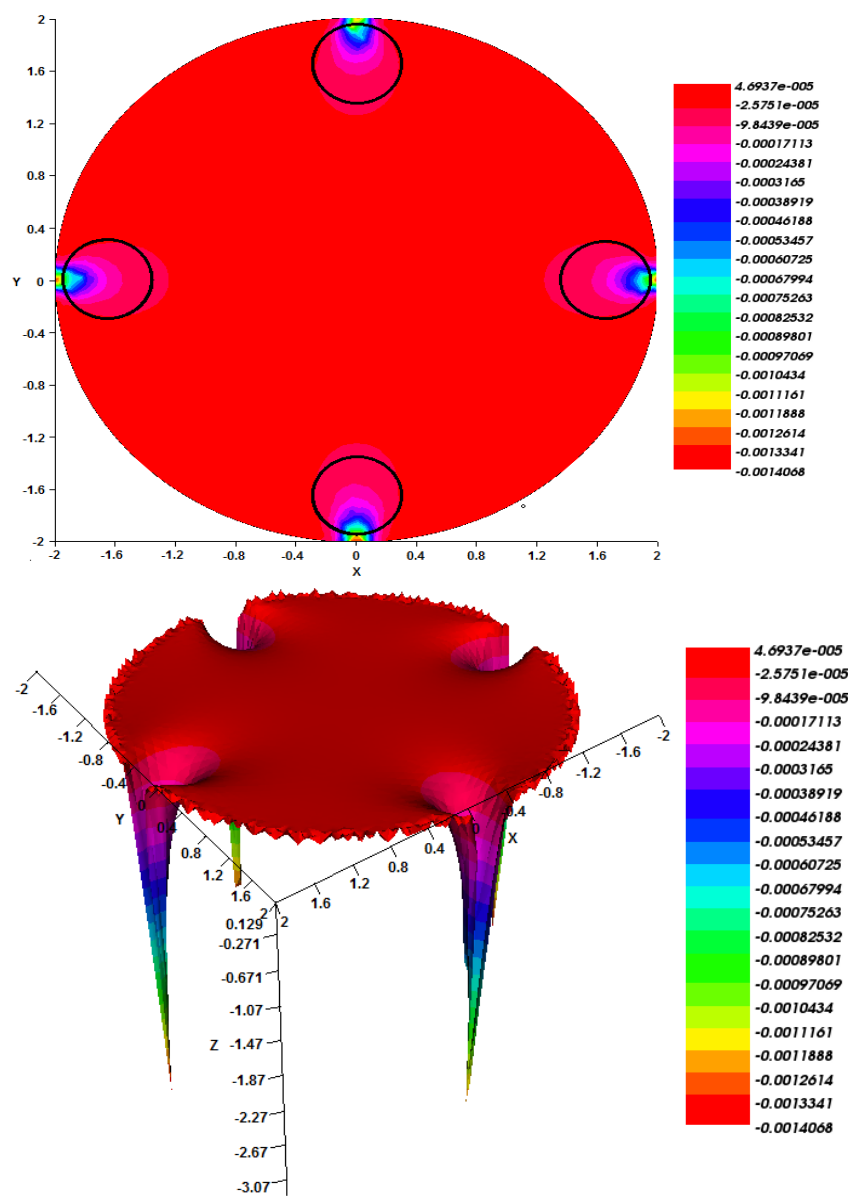

Figure 6: On the left: superposition of the actual cavities and negative leve lines of the topological gradient; on the right: the topological gradient $g$.

Verwendung von Lopchpositionierungkrieterien. Universität-Gesamthochschule Siegen, Siegen, Germany.

10. Garreau S, Guillaume P, Masmoudi M (2001) The topological asymptotic for PDE systems: The elasticity case. SIAM J Control Optim 39: 1756-1778.

11. Sokolowski J, Zochowski A (1999) On the topological derivative in shape optimization. SIAM J Control Optim 37: 1251-1272.

12. Ben Abda A, Hassine M, Jaoua M, Masmoudi M (2009) Topological sensitivity analysis for the location of small cavities in Stokes flow. SIAM J Control Optim 48: 2871-2900.

13. Caubet F, Dambrine M (2012) Localization of small obstacles in Stokes flow. Inverse Problems 28: 1-33.

14. Samet B, Amstutz S, Masmoudi M (2003) The Topological Asymptotic for the Helmholtz Equation. SIAM J Control Optim 42: 1523-1544.

15. Auroux D, Belaid LJ, Rjaibi B (2010) Application of the topological gradien method to color image restoration. SIAM J Imaging Sci 3: 153-175.

16. Belaid LJ, Jaoua M, Masmoudi M, Siala L (2008) Application of the topological gradient to image restoration and edge detection. Engineering Analysis with boundary Elements 32: 891-899.

17. Amstutz S (2003) Aspects théoriques et numériques en optimisation de forme topologique. Institut National des Sciences Appliquées de Toulouse.

18. Larnier S, Fehrenbach J, Masmoudi M (2012) The topological gradient method: From optimal design to image processing. Milan Journal of Mathematics 80 411-441.

19. Kohn R, Vogelius M (1987) Relaxation of a variational method for impedance computed tomography. Comm Pure Appl Math 40: 745-777. 
Citation: Jaiem E, Abda AB, Rjaibi B (2016) Cavities Identification from Partially Overdetermined Boundary Data in Linear Elasticity. J Appl Computat Math 5: 295. doi:10.4172/2168-9679.1000295

Page 9 of 9

20. Amstutz S, Horchani I, Masmoudi M (2005) Crack detection by the topological gradient method. Control and Cybernetics 34: 81-101.

21. Chaabane S, Masmoudi M, Meftahi H (2013) Topological and shape gradient strategy for solving geometrical inverse problems. J Math Anal Appl 400: 724742
22. Murat F, Simon J (1976) Sur le contrôle par un domaine géométrique. Université Pierre et Marie Curie, Paris.

23. Allaire G, Gournay F, Jouve F, Toader AM (2005) Structural optimization using topological and shape sensitivity via a level set method. Control and Cybernetics 34: 59-80. 